Physics of nuclei and elementary particles

Nuclei and Particles: An Introduction to Nuclear and Subnuclear Physics. By Emilio Segré. Pp. 966. (W. A. Benjamin: Reading, Massachusetts, 1977.) $\$ 29.50$.

THE original intention of this well known book, first published in 1964 and now in a second edition, remains unchanged, namely to provide potential research workers with a broad, but not superficial, introduction to the physics of nuclei and elementary particles, paying attention both to the theoretical aspects of the underlying physics and also to the experimental methods and techniques used in this field. The theoretical treatment is kept at the lowest possible level of sophistication necessary for a good understanding of the physical processes under discussion, and much healthy use is made of intuitive argument. Even so, a sound knowledge of quantum mechanics is expected of the reader and occasionally a familiarity with the elements of relativistic quantum mechanics.

Apart from a small introductory section and a set of appendices dealing with various technical matters, the book is divided into three main parts: "Tools", "The Nucleus" and "Particles”, occupying respectively 184, 469 and 239 of the total of 966 pages.

The first part is essentially in the same form as in the first edition, although thoroughly updated and with the inclusion of one or two additional subsections dealing with recent developments. There is a particularly fine and meaty chapter on the passage of radiations through matter, together with chapters on detection methods, particle accelerators (including a new section on colliding beams) and on the general features and applications of radioactive decay. Plenty (but not in excessive quantity) of technical detail is given and this part of the book, in particular, is written with masterly authority.

The discussion of what is generally called low energy nuclear physics in the second part of the book, covers the ground one would expect. There are large chapters on nuclear structure and nuclear reactions and smaller ones on $\alpha, \beta$ and $\gamma$ decay, the two-nucleon interaction, and neutron physics. Here, the balance and ordering of the matter dealt with might be questioned. For example, in a book of this size which sets out to be "introductory", to devote only seven pages (including three full pages of diagrams) to the nuclear shell model which, after all, underlies a lot of our thinking about the nucleus, seems rather parsimonious. It could also be argued that a discussion of the nuclear force itself and the two-nucleon system would better precede the treatment of nuclear forces in the nucleus, in particular, the role of the residual interaction and pairing effects. But these are minor points which hardly detract from what is an excellent treatment of the core of nuclear physics.

It is in the part of the book dealing with elementary particle physics that major differences between the two editions are to be found. This section has been completely rewritten and Professor Segré has now provided a wide, comprehensive and up-to-date account of the state of play in this field. After a general introductory chapter dealing with some general symmetry and conservation laws, he devotes succeeding chapters to leptons, pions, and other bosons and baryons. There follows a major chapter on the SU(3) classification of hadrons and quarks, with discussion of charm and colour. No attempt is made to deal with the mathematical details of the SU(3) group which are regarded (rightly) by the author as "beyond the scope of this book", a phrase which appears more than once in these last two hundred or so pages. The remaining three chapters deal with $\mathrm{e}^{+} / \mathrm{e}^{-}$collisions (including discussions of the $J / \psi$ particle), weak interaction physics (including unified theories) and CP violation, and high energy collisions of hadrons. Inevitably, because of the extreme mathematical complexity of much of the underlying theory, the treatment in this last part of the book has to be carried out at a more superficial level. Despite this limitation, the author has

\section{Nature of the seabed in a median valley}

Photographic Atlas of the Mid-Atlantic Ridge Rift Valley. By R. D. Ballard and J. G. Moore. Pp. 114. (Springer: New York and Berlin, 1978.) DM43.30; $\$ 21.70$.

IT is always difficult to publish for the benefit of one's fellow scientists observations that are entirely visual and very numerous. Ballard and Moore have produced an excellent book summarising the results of surveys by underwater photography, both from the submersible ALVIN and from the US Navy LIBEC System, which were made in support of the international study of accretion processes on the axis of the Mid-Atlantic Ridge, south-west of the managed most successfully to deal in an understandable way with a great many of the current ideas of elementary particle physics in line with his intention to introduce the reader to as many trees in the forest as possible.

Layout and typography are much to be preferred to those of the first edition, the layout of which was odd in the extreme. Particularly striking are the many diagrams, both illustrating apparatus and representing quantitative data in graphical form. Most are accompanied by excellent and full captions, and are extremely informative. As befits a pedagogical book, each chapter is accompanied by a set of problems which vary from simple numerical calculations to effective extensions of the subject matter of the chapter. In addition there is a general bibliography, a bibliography for each chapter and plentiful referencing to original papers.

The book is quite unique and constitutes a remarkable and readable exposition of most of the basic stuff of nuclear and particle physics ranging from details of experimental method through to advanced theoretical ideas. The whole is illuminated and characterised by the author's physical insight and admirably achieves his aim of conveying an intuitive understanding of the main phenomena of nuclear and particle physics to "physics students, chemists, and engineers who want to acquire enough knowledge of nuclear and subnuclear physics to be able to work in this field". Finally, it is not expensive for its size.

\section{R. J. Blin-Stoyle}

R. J. Blin-Stoyle is Professor of Theoretical Physics at the University of Sussex, $U K$.

Azores (Project FAMOUS). The features shown in the many thousand photographs taken, have been categorised into two classes: first, submarine volcanic products including the vents themselves, flow units and a great variety of pillow lavas and breccias; and second, faults, fissures and related tectonic features. As well as describing the geological setting, interpretations of the photographs are illustrated by excellent sketches and by diagrams showing the processes creating the microtopography.

I thoroughly recommend the book not only as a serious scientific record of the nature of the seabed in a median valley, but as an attractive and interesting book for the non-specialist.

\section{A. S. Laughton}

A. S. Laughton is Director of the Institute of Oceanographic Sciences (NERC), Wormley, UK. 